Research Paper

\title{
Polymeric immunoglobulin receptor (PIGR) exerts oncogenic functions via activating ribosome pathway in hepatocellular carcinoma
}

\author{
Yuan Zhang ${ }^{1 *}$, Jijie Zhang ${ }^{2 *}$, Xiaorong Chen ${ }^{1}$, Zongguo Yang ${ }^{1 凶}$ \\ 1. Department of Integrative Medicine, Shanghai Public Health Clinical Center, Fudan University, Shanghai 201508, China. \\ 2. Department of Oncology, The People's Hospital of Danyang, Affiliated Danyang Hospital of Nantong University, Jiangsu 212300, China. \\ *These authors contributed equally to this work. \\ $\triangle$ Corresponding authors: Zongguo Yang, M.D., Ph.D., Shanghai Public Health Clinical Center, Fudan University. 2901 Caolang Road, Shanghai 201508, China. \\ E-mail: yangzongguo@shphc.org.cn. \\ (C) The author(s). This is an open access article distributed under the terms of the Creative Commons Attribution License (https://creativecommons.org/licenses/by/4.0/). \\ See http://ivyspring.com/terms for full terms and conditions.
}

Received: 2020.06.22; Accepted: 2020.11.03; Published: 2021.01.01

\begin{abstract}
Objective: This report aimed to investigate the potential mechanism of polymeric immunoglobulin receptor (PIGR) in promoting cancer development in hepatocellular carcinoma (HCC).

Methods: PIGR expression was investigated in Gene Expression Omnibus (GEO), Oncomine, The Cancer Genome Atlas (TCGA) and The Human Protein Atlas (HPA) databases. Relationships between PIGR and HCC survival and clinico-pathological features were conducted in TCGA. RNAseq of PIGR overexpression and knockdown samples in Bel-7404 cells were performed for identifying potential mechanisms.

Results: PIGR was significantly overexpressed in tumors compared to nontumors and in HCC serum peripheral blood mononuclear cells (PBMC) than in healthy individuals (all $p<0.05$ ). In TCGA, PIGR was highly altered in 14\% HCC patients. PIGR upregulation was significantly associated with poor disease-free survival $(p<0.05)$. More patients recurred/progressed in PIGR altered group compared to unaltered group $(p<0.01)$. PIGR was significantly higher in HCC patients with incomplete cirrhosis $(p<0.001)$ and established cirrhosis $(p<0.05)$. Fewer patients had $N_{0}$ lymph node stage in PIGR altered group than those in the unaltered group $(p<0.05)$. PIGR RNAseq revealed that ribosome signaling was the common pathway in PIGR overexpression and PIGR knockdown samples. RNAseq analysis indicated that RPL10, RPL10A, RPL12, RPL19, RPL36, RPL38, RPL41, RPL6, RPL8, RPS12, RPS14, RPS15A, RPS2, RPS27A and RPSA were significantly upregulated in PIGR overexpression group and downregulated in PIGR underexpression group (all $p<0.05$ ).
\end{abstract}

Conclusions: Aberrant PIGR was associated with HCC recurrence, and PIGR stimulated ribosome pathway might be a potential mechanism.

Key words: polymeric immunoglobulin receptor; hepatocellular carcinoma; disease-free survival; recurrence; ribosome

\section{Introduction}

Liver cancer, comprising $75 \% \sim 85 \%$ cases of hepatocellular carcinoma (HCC), is predicted to be the sixth common and the fourth lethal cancer worldwide [1-3]. During the past two decades, there has been a marked increase in HCC incidence and cancer related annual deaths $[3,4]$. A series of complex multistep hepatocarcinogenesis mechanisms of angiogenesis, invasion, metastasis, regulation of cell cycle, proliferation, differentiation, cell invasion, and inflammation were involved in HCC progression [5, 6]. However, the details and mechanisms of hepatocarcinogenesis still remain to be elucidated.

Solely produced by intestinal epithelial cells, polymeric immunoglobulin receptor (PIGR) captured 
and transcytosed dimeric $\operatorname{Ig} \mathrm{A}(\mathrm{d} \operatorname{Ig} \mathrm{A})$ from lamina propria to intestinal lumen across epithelial cells, participating in mucosal immune system $[7,8]$. Previous evidence indicated that proinflammation cytokines could activate Janus kinase-signal transduction and activator of transcription (JAKSTAT), NF-KB and mitogen-activated protein kinase (MAPK) signaling pathways, leading to PIGR overexpression [7, 8]. Moreover, downregulation of PIGR resulted in inhibition of cell proliferation, adhesion and migration in pancreatic ductal adenocarcinoma (PDAC) [9]. Recently, PIGR has been proved to be involved in the human tumorigenesis and malignancies [9-12]. As a vital inflammatory mediator [7], PIGR played an important role in hepatitis B virus (HBV) infection, chronic liver inflammation, tumor growth, recurrence, and metastatic progression in HCC [10-12]. Mechanistically, Smad induced epithelialmesenchymal transition (EMT) [10] and Rac1/ CDC42-MEK/ERK cascade [12] were proved to be potential mechanisms of PIGR related cancer malignancy in HCC.

As part of intestinal immune network for $\operatorname{Ig} A$ production pathway, we previously found that PIGR was overexpressed in HCC tumors [13]. In this analysis, we aimed to investigate the oncogenic functions of PIGR and the potential mechanism of PIGR in promoting malignancy in HCC.

\section{Materials and Methods}

\section{Ethical Statement}

Our study did not require an ethical board approval because it did not contain human or animal trials.

\section{Data sources}

Studies compared PIGR between cancer and normal samples in liver cancer were selected with analysis type by "liver cancer vs. normal", gene name by "PIGR", threshold by $p$-value $\leq 1 \mathrm{E}-4$, fold change $\geq$ 2 and top $10 \%$ gene rank in Oncomine database (https://www.oncomine.org/). Profiles including Guichard Liver, Guichard Liver 2 and TCGA liver were included in this analysis. In order to validate the PIGR expression levels in serum and tissues HCC and healthy candidates, Gene Expression Omnibus (GEO, https://www.ncbi.nlm.nih.gov/geo/) database was searched using heading terms including "liver cancer" and "hepatocellular carcinoma". All the series with expression profiling by array were included and homo sapiens were selected. Platforms with terms including "ID", "Gene Symbol" and "ENTREZ_GENE_ID" were also restricted. GEO profiles including GSE49515 [14], GSE55092 [15] and
GSE60502 [16] were included in this analysis. Additionally, PIGR protein detected by immunohistochemistry was obtained from the Human Protein Atlas (HPA) databases (http://www.proteinatlas. org) [17-19].

\section{Bioinformatics analysis}

Raw.CEL files of the microarray from each GEO dataset were normalized by quantile method of Robust Multichip Analysis (RMA) from $R$ affy package [20]. Gene expression of PIGR between tumor and nontumor tissues was performed by $\mathrm{R}$ Limma package [21]. Series datasets with expression profiling by array was searched by gene name "PIGR" in GEO database, and GSE34630 [22] profile was used for gene set enrichment analysis (GSEA) by GSEA software [23].

\section{Cell culture}

Bel-7404 human HCC cell line was purchased from Chinese Academy of Sciences. Cells were cultured in Dulbecco's modified Eagles' medium (DMEM/high glucose) supplemented with $10 \%$ fetal bovine serum (FBS) (Cellsera, NSW, Australia) containing $50 \mu \mathrm{g} / \mathrm{ml}$ penicillin and $50 \mu \mathrm{g} / \mathrm{ml}$ streptomycin at $37^{\circ} \mathrm{C}$ in a $5 \% \mathrm{CO} 2$ incubator. The medium was changed three times every week.

\section{Vector construction and transduction}

A lentiviral PIGR short hairpin RNA (shRNA) was purchased from Genechem (Shanghai, China). The shRNA sequence targeting human PIGR complementary DNA were as follows:

- shRNA1-PIGR: TCGATCACTCAGGAGACAT;

- shRNA2-PIGR: CGTCTATGTGGCAGTTGAA;

- shRNA3-PIGR: CAACTATACAGGAAGAATA.

A scrambled shRNA was included as a negative control (NC). The PIGR overexpression plasmid was generated from human total cDNA. This construct was generated with the forward and reverse primers. Forward: 5' GAGGATCCCCGGGTACCGGTCGCCA CCATGGCGGAGCCGAGCGGC3', reverse: 5' TCAC CATGGTGGCGACCGGGCTGACACTCAACTGAG CA3'. The target sequence was inserted into GV248 lentiviral vector (Genechem, Shanghai).

\section{Transcriptome sequencing analysis}

To identify mRNAs in Bel-7404 cells in response to PIGR challenges, mRNA libraries derived from three groups including PIGR overexpression group, PIGR knockdown group and negative control groups were constructed and sequenced using the Illumina Novaseq6000-sequencing platform. The mRNA sequencing analysis was conducted by Novogene (Tianjin, China). 


\section{Quantitative real-time PCR (qRT-PCR)}

Expression levels of genes screened by transcriptome sequencing analysis were verified by qRT-PCR according to the manufacturer's instructions of Takara (Takara Bio Inc, Shiga, Japan). The primer sequence of these genes for qRT-PCR was summarized in Table 1.

\section{Western blotting analysis}

Western blot analysis was performed according to standard procedures using antibodies against PIGR (ImmunoWay Biotechnology, TX, USA) and actin (Cell Signaling Technology, MA, USA). The protein expression detected by western blot were analyzed and quantified using Image J software (https:// imagej.nih.gov/ij/index.html).

\section{Survival analysis}

Liver Hepatocellular Carcinoma (TCGA, Firehose Legacy) database in cBioPortal for cancer genomics web service was obtained [24, 25]. PIGR mRNA expression levels calculated by $\log 2$ calculation were compared based on clinical attribute in HCC patients. To evaluate associations between PIGR and survival and clinic-pathological features in HCC patients, gene data with $\mathrm{Z}$ scores and clinical data of HCC patients in Liver Hepatocellular Carcinoma (TCGA, Firehose Legacy) database were downloaded from cBioPortal and matched using VLOOKUP index in EXCEL. According to the pathological diagnosis, 361 HCC patients were included in the analysis.

\section{Statistical analysis}

Differences of variables between the individual groups were analyzed using student $t$ test, MannWhitney $U$-test and Chi-square test based on variables types. The Kaplan-Meier method with log rank test was used to compare survival between different groups. Stata software version 16.0 (Stata Corp LLC, Texas, USA) was used. A two-tailed $p<0.05$ were considered significant for all tests.

\section{Results}

\section{PIGR expression levels between tumors and nontumors}

As shown in Figure 1, PIGR mRNA was significantly upregulated in tumor tissues compared to nontumor tissues in Guichard Liver, Guichard Liver 2 and TCGA Liver from Oncomine database (all $p<0.0001$, Figure 1A), as well as in GSE55092 and
(A)

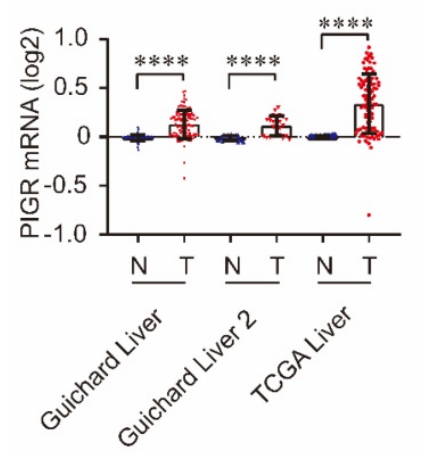

(B)

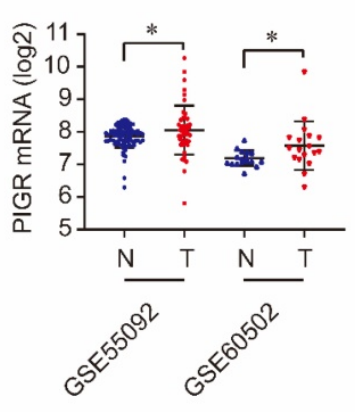

(C)

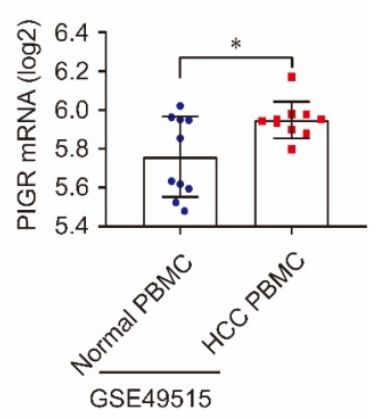

(D)

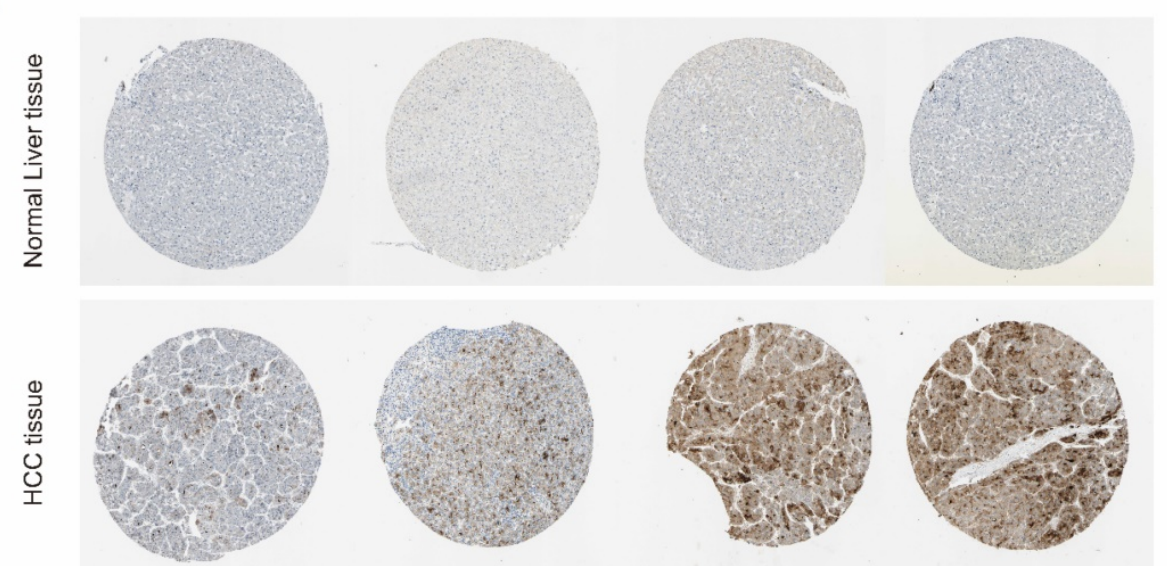

Figure 1. PIGR expression in HCC tissues and serum samples. PIGR mRNA was significantly upregulated in Guichard Liver, Guichard Liver 2 and TCGA Liver in Oncomine database (A), and in GSE55092 and GSE60502 in GEO database (B); PIGR mRNA was significantly overexpressed in HCC PBMC samples compared to that in healthy individuals (C); PIGR protein was highly expressed in HCC tumor tissues compared to that in normal liver tissues (D).* $p<0.05$, *** $p<0.01$, **** $p<0.001, * * * * p<0.0001$. 
GSE60502 profiles in GEO databases (both $p<0.05$, Figure 1B). In serum samples, PIGR mRNA was significantly overexpressed in PBMC of HCC patients compared to that in healthy individuals $(p<0.05$, Figure 1C). We also detected the PIGR protein levels by immunohistochemistry in HPA database. As shown in Figure 1D, PIGR protein was higher in tumor tissues than nontumor tissues in HCC patients, in consistent with our previous report [13].

\section{Associations between PIGR and survival and clinico-pathological features}

In cBioPortal database, PIGR mRNA was upregulated in 14\% (51/371) patients (Figure 2A). Patients without PIGR expression data were excluded, and 306 patients were included in the survival analysis. As shown in Figure 2B, high PIGR was significantly associated with worse disease-free survival (DFS) in HCC patients ( $p=0.043$, Figure 2B). In PIGR altered group, more patients developed recurrence/progression than those in unaltered group (76.2\% vs. $51.4 \%, p<0.01$, Figure 2 C). PIGR was significantly higher in HCC patients with nodular formation and incomplete cirrhosis $(p<0.001$, Figure 2D) and established cirrhosis $(p<0.05$, Figure 2D). Fewer patients had $\mathrm{N}_{0}$ lymph node stage in PIGR altered group than those in unaltered group by American Joint Committee on Cancer code $(p<0.05$, Figure 2E).

(A)

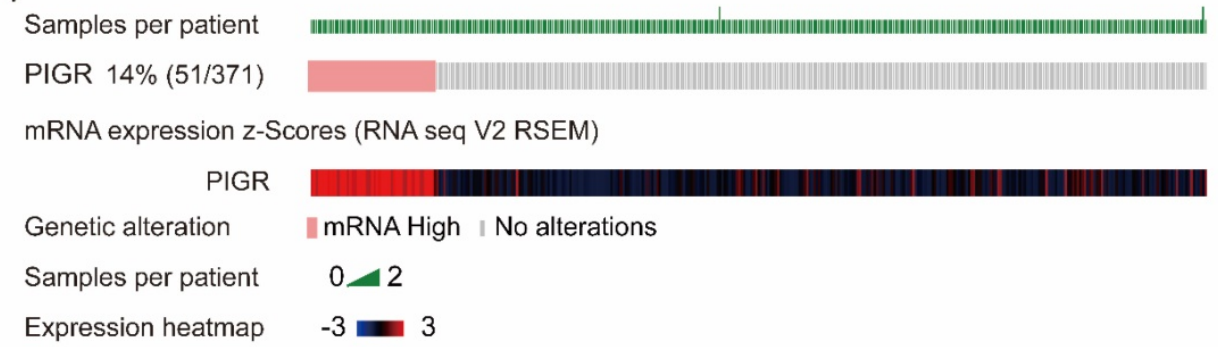

(B)

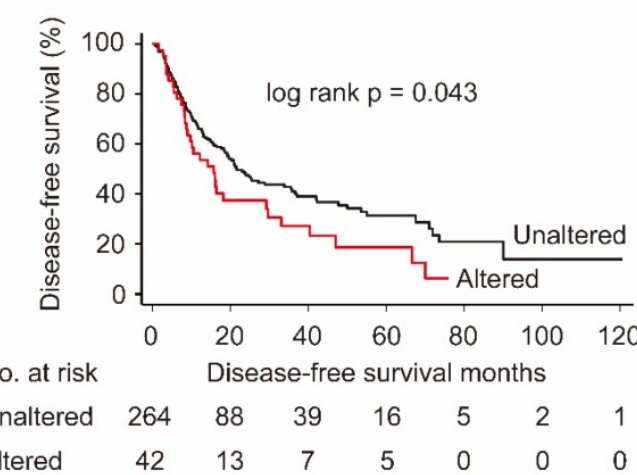

(D)

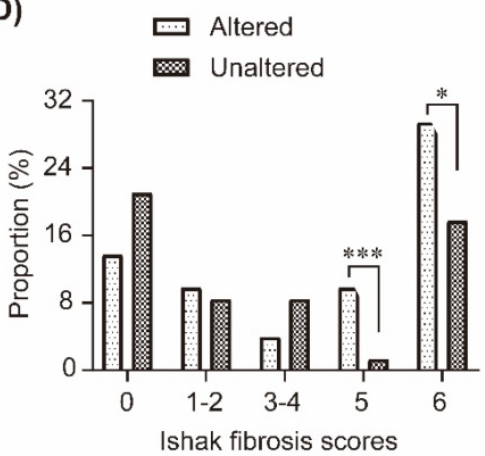

(C)

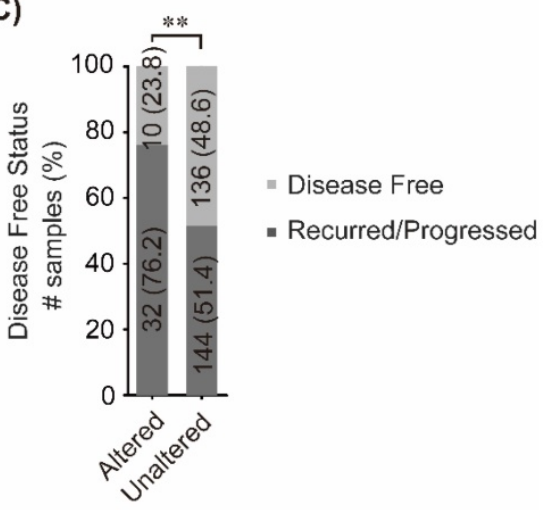

(E)

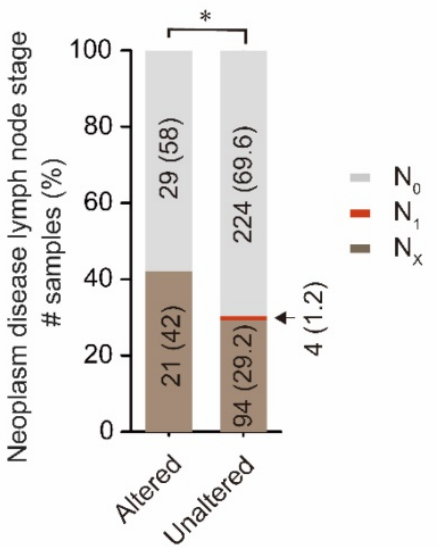

Figure 2. Associations between PIGR and survival and clinico-pathological features in HCC patients from cBioPortal database. $14 \%$ (51/371) HCC patients had PIGR highly altered in tumor tissues (A); High PIGR mRNA was significantly associated with worse disease-free survival in HCC patients (B); More patients developed recurrence/progression in PIGR altered group than those in unaltered group (C); PIGR was significantly higher in HCC patients with nodular formation and incomplete cirrhosis and established cirrhosis (D); Less patients had $\mathrm{N}_{0}$ lymph node stage in PIGR altered group than those in unaltered group by American Joint Committee on Cancer code (E). * $p<0.05, * * p<0.01, * * * p<0.001, * * * * p<0.0001$. 
Table 1. Primer sequence of genes screened by transcriptome sequencing analysis

\begin{tabular}{lll}
\hline Gene & Forward & Reverse \\
\hline RPL10 & GATGCCAAGATTCGCATTTTTG & AGCTGCTCATATTCATCTGACA \\
RPL10A & GATCAAGCAGATTCCACGAATC & GAACTTGATTGTGGACTTCACC \\
RPL12 & ACCACCAAGAGACAGAAAGAAA & CGAGCAATGTTGACAATCTCAT \\
RPL19 & TCACATGGATGAGGAGAATGAG & CGCTTGTTTTGAACACATTCC \\
RPL36 & CAAAGTGACCAAGAACGTGAG & TCCCCACCCTTTTCTTGATAAA \\
RPL38 & GGATGCCAAATCTGTCAAGATC & TTCTCTTTGTCAGTGATGACCA \\
RPL41 & GCCGTAGACGGAACTTCGCC & TCTGCTCCTGTGGCCTCCAC \\
RPL6 & CATGTATTCCAGAAAGGCCATG & GCATTTGCGAAGTTTAACCAC \\
RPL8 & TTTGTGTATGCGGCAAGAAG & GAGATAACGGTGGCATAGTTCC \\
RPS12 & CCAACTGTGATGAGCCTATGTA & TTTACAAAGGCCTACCCATTCT \\
RPS14 & GAGAATGTATTTGGTGTTGCC & GTTTCCTTGCCAGAAAGATCAG \\
RPS15A & CTCACTGTGATGATGAAGCATG & AGTACAATGAAACCAAACTGGC \\
RPS2 & GCCAAGCTCTCCATCGTC & GTGCAGGGATGAGGCGTA \\
RPS27A & CTGGAAGATGGACGTACTTTGTC & CGACGAAGGCGACTAATTTGC \\
RPSA & GTGGCACCAATCTTGACTTCC & GCAGGGTTTTCAATGGCAACAA \\
\hline
\end{tabular}

(A)

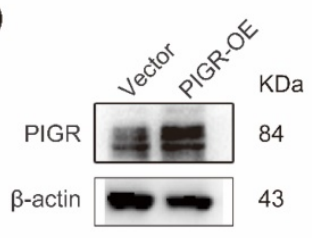

(C)

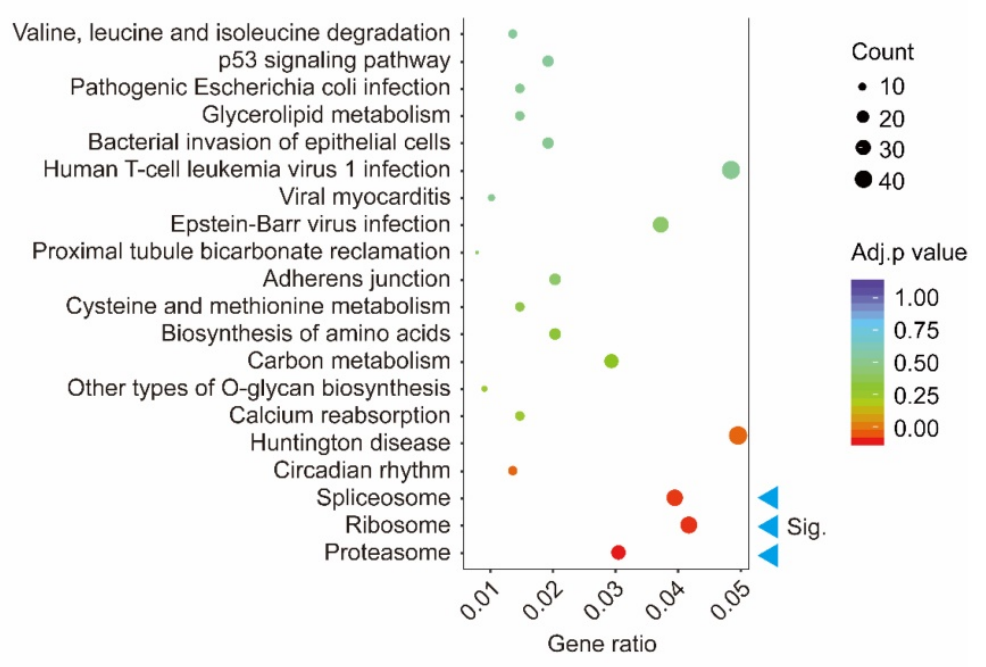

(D)

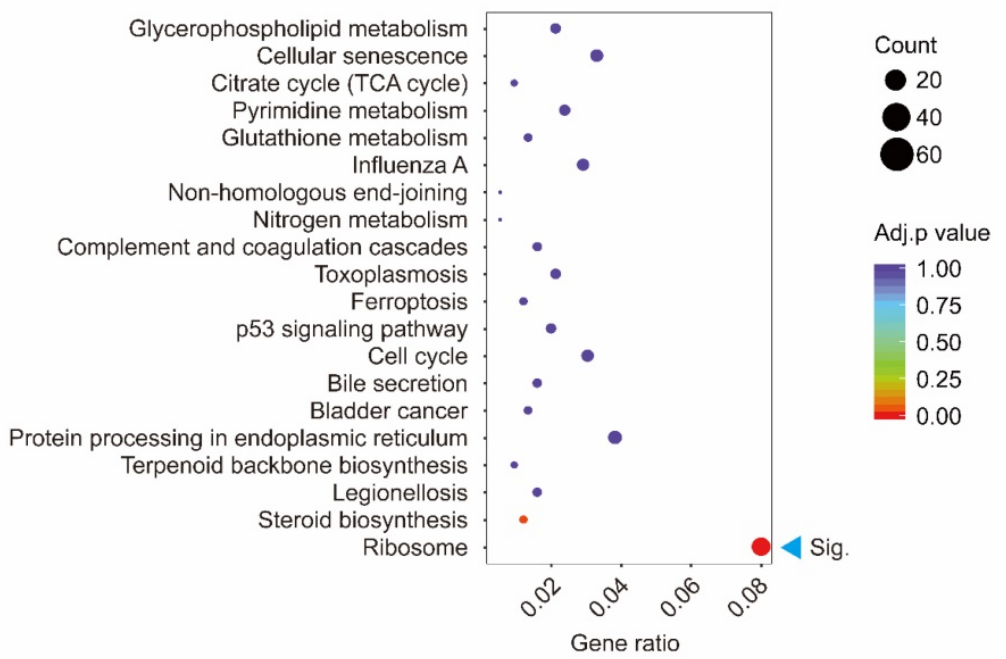

Figure 3. KEGG enrichment of genes changed with PIGR expression. The overexpression efficiency and knockdown efficiency of PIGR (A); Three pathway including spliceosome, ribosome and proteasome were significantly enriched in PIGR overexpression group (B); Ribosome pathway was significantly enriched in PIGR knockdown group (C). ${ }^{*} p<0.05, * * p<0.01, * * * p<0.001, * * * * p<0.0001$. 
(A)

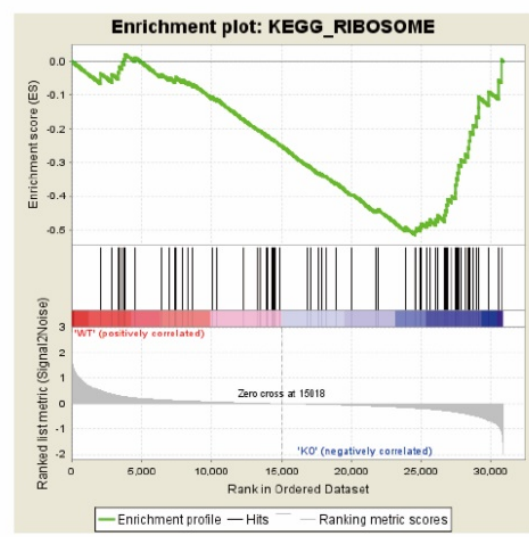

(C)

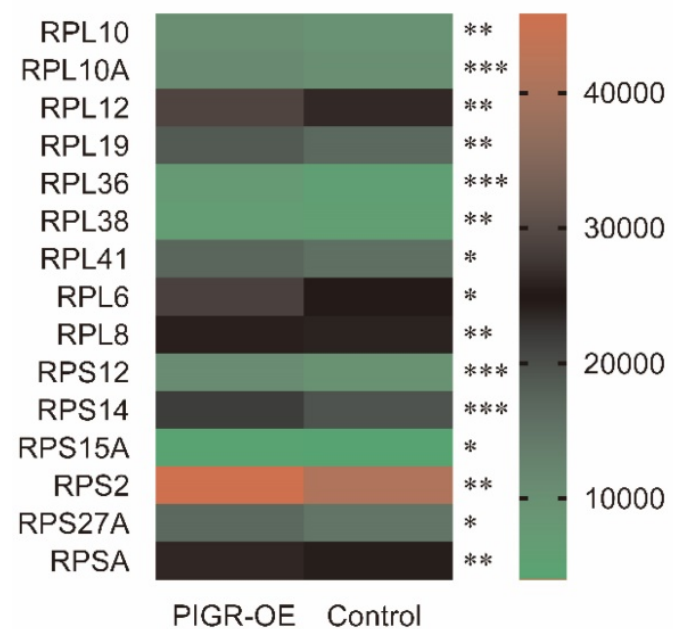

(B)

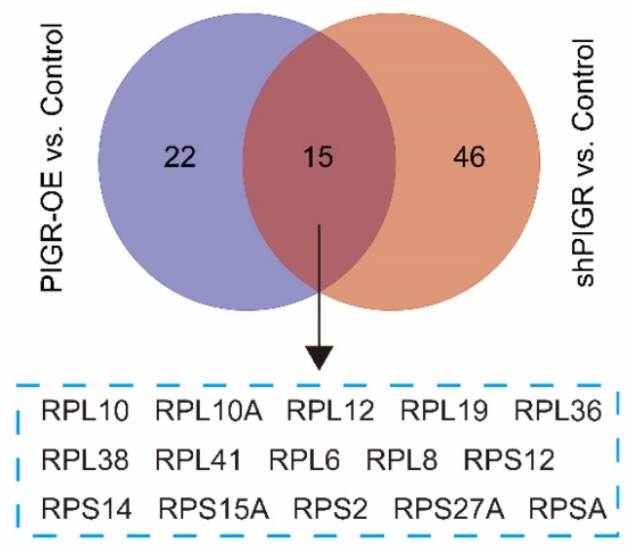

(D)

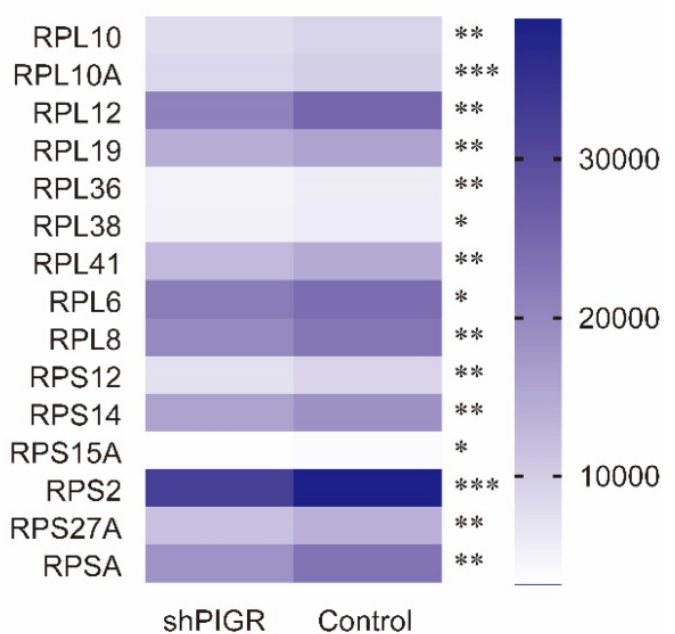

Figure 4. Common genes changed with PIGR expression. In GSE34630, wild type mice left untreated (5 replicates) and PIGR knockout mice left untreated (6 replicates) were included for GSEA enrichment. Ribosome pathway was one potential pathway induced by PIGR (A). According to the RNAseq results, 15 genes were common genes both in PIGR overexpression and knockdown groups compared to negative control group (B). All these 15 genes were significantly overexpressed in PIGR overexpression group (C), and downregulated in PIGR knockdown group (D). * $p<0.05$, ** $p<0.01$, *** $p<0.001$, **** $p<0.0001$.

\section{PIGR activated ribosome pathway}

We performed KEGG pathway enrichment in PIGR overexpression group and PIGR knockdown group using transcriptome sequencing analysis. The overexpression efficiency and knockdown efficiency of PIGR were shown in Figure 3A and 3B. The most effective short hairpin RNA (shRNA1-PIGR) of PIGR knockdown was selected for the transcriptome sequencing analysis (Figure 3B). Three pathway including spliceosome, ribosome and proteasome were significantly enriched in PIGR overexpression group (Figure 3C), while only ribosome pathway was significantly enriched in PIGR knockdown group (Figure 3D). In GSE34630, wild type mice left untreated (5 replicates) and PIGR knockout mice left untreated (6 replicates) were included for GSEA enrichment. As shown in Figure 4, ribosome pathway was one potential pathway induced by PIGR (Figure 4A). According to our RNAseq results, 15 genes including RPL10, RPL10A, RPL12, RPL19, RPL36, RPL38, RPL41, RPL6, RPL8, RPS12, RPS14, RPS15A, RPS2, RPS27A and RPSA were common genes both in PIGR overexpression and knockdown groups compared to negative control group (Figure 4B). All these 15 genes were significantly overexpressed in PIGR overexpression group (all $p<0.05$, Figure 4 C), and downregulated in PIGR knockdown group (all $p$ $<0.05$, Figure 4D).

In addition, we conducted qRT-PCR analysis to verify expression levels of these 15 genes. As summarized in Figure 5, RPL10, RPL10A, RPL12, RPL19, RPL36, RPL6, RPL8, RPS12, RPS14, RPS15A, RPS2, RPS27A and RPSA were all upregulated in PIGR overexpression group compared to those in negative control group (all $p<0.05$, Figure 5). While RPL10, RPL10A, RPL19, RPL38, RPL6, RPL8, RPS12, RPS15A, RPS2 and RPS27A were all downregulated in PIGR knockdown group than those in negative control group (all $p<0.05$, Figure 5). 


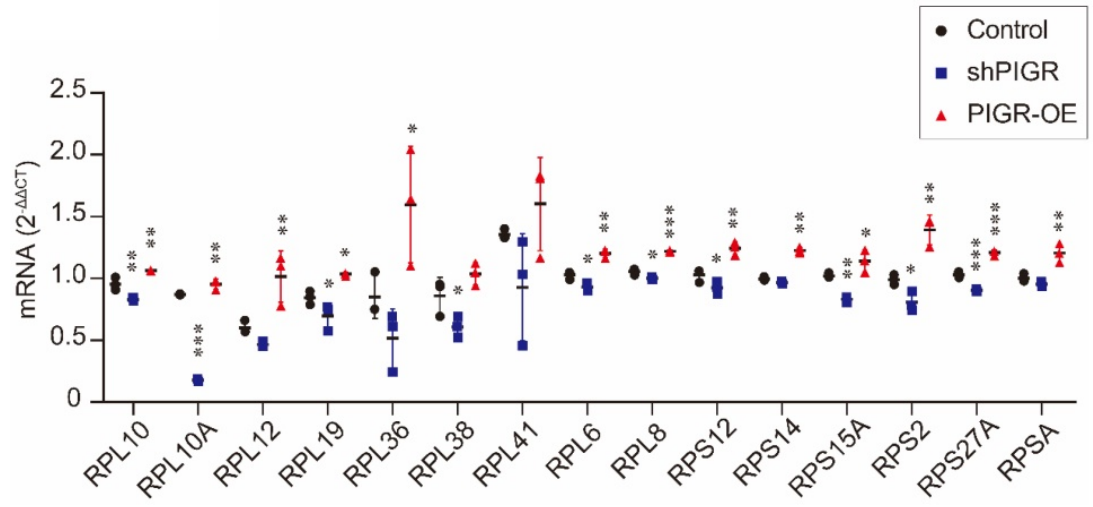

Figure 5. Expression levels of screened genes by RNAseq using qRT-PCR analysis. $* p<0.05, * * p<0.01, * * * p<0.001, * * * * p<0.0001$.

\section{Discussion}

In line with previous reports [10, 13], we found that PIGR was upregulated in tumor tissues and serum samples in HCC patients. In addition, high PIGR contributed to advanced liver fibrosis stage, low incidence of lymph node stage $\mathrm{N}_{0}$, and high risk of recurrence. Considered previous literatures and our results, we assumed that PIGR exerts as an oncogene in the development of HCC.

The extracellular portion of PIGR binding to dIgA is called the secretory component (SC), which is responsible for intracellular neutralization of some viruses [26, 27]. Cytokines released by host and adaptive immune cells including interferon (IFN)- $\gamma$, interleukin (IL)-1, IL-4, IL-17, tumor necrosis factor (TNF) and lymphotoxin (LT)- $\beta$ could also indirectly simulating the PIGR expression [28-30]. Consequently, these cytokines involved in multiple intracellular signaling pathway including JAK-STAT, NF-KB and MAPK, resulting in upregulation of PIGR $[7,8]$. In PDAC, downregulation of PIGR results in reduction of cellular proliferation, adhesion and migration in vitro [9]. In vivo, PIGR downregulation resulted in reduced cancer cell invasion and diminished stromal activity. And, low PIGR correlated with better survival in PDAC patients [9]. Additionally, chronic inflammation induced by HBV might upregulate the expression of PIGR. PIGR upregulation increased the nuclear translocation of Smad2/3, leading to the activation of Smad pathway. Consequently, the transcriptional regulation mediated by Snail, Slug and ZEB1 leads to promotion of EMT $[10,11]$. All these underlying mechanisms supported the hypothesis that PIGR exerts an oncogenic function in the hepatocarcinogenesis.

Even though, the downstream signaling of PIGR remains to be elucidated. According to our results, we believed that PIGR might simulate ribosome pathway activation and promoted cancer progression. Ribosomes are required for protein production, and are involved in the process of cell proliferation, growth and survival [31]. Current evidences have elucidated that inhibition of ribosome biogenesis could induce p53 stabilization and activation, mainly through ribosomal proteins (RPs)-MDM2-p53 pathway [32-34]. Recently, ribosome biogenesis has emerged as an effective target in cancer therapy. A series of compounds that inhibit ribosome biosynthesis or function have shown their toxic action on cancer chemotherapy [35-37]. Recent literatures of HCC ribosome profiling also provided insightful data resource for dissecting the translatome shift in liver cancer, at sub-codon resolution, and the regulatory mechanisms of oncogenic signaling and HCC therapy [38-40]. In our study, we demonstrated that ribosome was the vital signaling pathway in accordance to PIGR expression changes. Ribosome genes changed in line with PIGR in vitro. Unfortunately, we did not perform experiments in vivo and potential connections between PIGR and ribosome protein still needs to be investigated.

Considered the critical roles of PIGR and ribosome in the development of human malignancies, we cautiously assumed that PIGR might exert oncogenic functions through simulating activation of ribosome pathway in the progression of HCC.

\section{Acknowledgements}

\section{Funding}

This work was supported by National Natural Science Foundation of China (81803901).

\section{Data availability}

Datasets of the current study are available from the corresponding author on reasonable request.

\section{Ethical Statement}

Our study did not require an ethical board approval because it did not contain human or animal trials. 


\section{Competing Interests}

The authors have declared that no competing interest exists.

\section{References}

1. Bray F, Ferlay J, Soerjomataram I, et al. Global cancer statistics 2018: GLOBOCAN estimates of incidence and mortality worldwide for 36 cancers in 185 countries. CA Cancer J Clin. 2018; 68: 394-424.

2. Heimbach JK, Kulik LM, Finn RS, et al. AASLD guidelines for the treatment of hepatocellular carcinoma. Hepatology. 2018; 67: 358-80.

3. Omata M, Cheng AL, Kokudo N, et al. Asia-Pacific clinical practice guidelines on the management of hepatocellular carcinoma: a 2017 update. Hepatol Int. 2017; 11: 317-70.

4. Petrick JL, Kelly SP, Altekruse SF, et al. Future of Hepatocellular Carcinoma Incidence in the United States Forecast Through 2030. J Clin Oncol. 2016; 34: 1787-94

5. Hanahan D, Weinberg RA. Hallmarks of cancer: the next generation. Cell. 2011; 144: 646-74.

6. Yuksel S, Boylu Akyerli C, Cengiz Yakicier M. Angiogenesis, Invasion, and Metastasis Characteristics of Hepatocellular Carcinoma. J Gastrointest Cancer. 2017; 48: 256-9.

7. Asano M, Komiyama K. Polymeric immunoglobulin receptor. J Oral Sci. 2011; 53: $147-56$

8. Johansen FE, Kaetzel CS. Regulation of the polymeric immunoglobulin receptor and IgA transport: new advances in environmental factors that stimulate $\mathrm{pIgR}$ expression and its role in mucosal immunity. Mucosal Immunol. 2011; 4: 598-602

9. Arumugam P, Bhattacharya S, Chin-Aleong J, et al. Expression of polymeric immunoglobulin receptor and stromal activity in pancreatic ductal adenocarcinoma. Pancreatology. 2017; 17: 295-302.

10. Ai J, Tang $\mathrm{Q}, \mathrm{Wu} \mathrm{Y}$, et al. The role of polymeric immunoglobulin receptor in inflammation-induced tumor metastasis of human hepatocellular carcinoma. J Natl Cancer Inst. 2011; 103: 1696-712.

11. Sphyris N, Mani SA. pIgR: frenemy of inflammation, EMT, and HCC progression. J Natl Cancer Inst. 2011; 103: 1644-5.

12. Yue $\mathrm{X}, \mathrm{Ai} \mathrm{J}, \mathrm{Xu} \mathrm{Y}$, et al. Polymeric immunoglobulin receptor promotes tumor growth in hepatocellular carcinoma. Hepatology. 2017; 65: 1948-62.

13. Yang Z, Tao Y, Xu X, et al. Bufalin inhibits cell proliferation and migration of hepatocellular carcinoma cells via APOBEC3F induced intestinal immune network for IgA production signaling pathway. Biochem Biophys Res Commun. 2018; 503: 2124-31

14. Shi M, Chen MS, Sekar K, et al. A blood-based three-gene signature for the non-invasive detection of early human hepatocellular carcinoma. Eur J Cancer. 2014; 50: 928-36

15. Melis M, Diaz G, Kleiner DE, et al. Viral expression and molecular profiling in liver tissue versus microdissected hepatocytes in hepatitis B virus-associated hepatocellular carcinoma. J Transl Med. 2014; 12: 230.

16. Wang YH, Cheng TY, Chen TY, et al. Plasmalemmal Vesicle Associated Protein (PLVAP) as a therapeutic target for treatment of hepatocellular carcinoma. BMC Cancer. 2014; 14: 815.

17. Thul PJ, Akesson L, Wiking M, et al. A subcellular map of the human proteome. Science. 2017; 356: eaal3321.

18. Uhlen M, Fagerberg L, Hallstrom BM, et al. Proteomics. Tissue-based map of the human proteome. Science. 2015; 347: 1260419.

19. Uhlen $M$, Zhang $C$, Lee $S$, et al. A pathology atlas of the human cancer transcriptome. Science. 2017; 357: eaan2507.

20. Gautier L, Cope L, Bolstad BM, et al. affy--analysis of Affymetrix GeneChip data at the probe level. Bioinformatics. 2004; 20: 307-15.

21. Ritchie ME, Phipson B, Wu D, et al. limma powers differential expression analyses for RNA-sequencing and microarray studies. Nucleic Acids Res. 2015; 43: e47.

22. Reikvam DH, Derrien M, Islam R, et al. Epithelial-microbial crosstalk in polymeric Ig receptor deficient mice. Eur J Immunol. 2012; 42: 2959-70.

23. Subramanian A, Tamayo P, Mootha VK, et al. Gene set enrichment analysis: a knowledge-based approach for interpreting genome-wide expression profiles. Proc Natl Acad Sci US A. 2005; 102: 15545-50.

24. Cerami E, Gao J, Dogrusoz U, et al. The cBio cancer genomics portal: an open platform for exploring multidimensional cancer genomics data. Cancer Discov. 2012; 2: 401-4.

25. Gao J, Aksoy BA, Dogrusoz U, et al. Integrative analysis of complex cancer genomics and clinical profiles using the cBioPortal. Sci Signal. 2013; 6: pl1.

26. Bomsel M, Heyman M, Hocini H, et al. Intracellular neutralization of HIV transcytosis across tight epithelial barriers by anti-HIV envelope protein dIgA or IgM. Immunity. 1998; 9: 277-87.

27. Yan H, Lamm ME, Bjorling E, et al. Multiple functions of immunoglobulin A in mucosal defense against viruses: an in vitro measles virus model. J Virol. 2002; 76: 10972-9.

28. Chintalacharuvu KR, Morrison SL. Production of secretory immunoglobulin A by a single mammalian cell. Proc Natl Acad Sci U S A. 1997; 94: 6364-8.
29. Crottet $\mathrm{P}$, Corthesy B. Secretory component delays the conversion of secretory IgA into antigen-binding competent $\mathrm{F}\left(\mathrm{ab}^{\prime}\right) 2$ : a possible implication for mucosal defense. J Immunol. 1998; 161: 5445-53.

30. Kaetzel CS. Cooperativity among secretory $\operatorname{IgA}$, the polymeric immunoglobulin receptor, and the gut microbiota promotes host-microbial mutualism. Immunol Lett. 2014; 162: 10-21.

31. Turi Z, Lacey M, Mistrik M, et al. Impaired ribosome biogenesis: mechanisms and relevance to cancer and aging. Aging (Albany NY). 2019; 11: 2512-40.

32. Dai MS, Lu H. Inhibition of MDM2-mediated p53 ubiquitination and degradation by ribosomal protein L5. J Biol Chem. 2004; 279: 44475-82.

33. Lohrum MA, Ludwig RL, Kubbutat MH, et al. Regulation of HDM2 activity by the ribosomal protein L11. Cancer Cell. 2003; 3: 577-87.

34. Zhang Y, Wolf GW, Bhat K, et al. Ribosomal protein L11 negatively regulates oncoprotein MDM2 and mediates a p53-dependent ribosomal-stress checkpoint pathway. Mol Cell Biol. 2003; 23: 8902-12.

35. Brighenti E, Trere D, Derenzini M. Targeted cancer therapy with ribosome biogenesis inhibitors: a real possibility? Oncotarget. 2015; 6: 38617-27.

36. Catez F, Dalla Venezia N, Marcel V, et al. Ribosome biogenesis: An emerging druggable pathway for cancer therapeutics. Biochem Pharmacol. 2019; 159: 74-81.

37. Penzo M, Montanaro L, Trere D, et al. The Ribosome Biogenesis-Cancer Connection. Cells. 2019; 8:55.

38. Wang $\mathrm{YH}$, Huang S, Zhu L, et al. Alternative transcription start site selection in ACSS2 controls its nuclear localization and promotes ribosome biosynthesis in hepatocellular carcinoma. Biochem Biophys Res Commun. 2019; 514: 632-8.

39. Xu W, Deng B, Lin P, et al. Ribosome profiling analysis identified a KRAS-interacting microprotein that represses oncogenic signaling in hepatocellular carcinoma cells. Sci China Life Sci. 2020; 63: 529-42.

40. Zou Q, Xiao Z, Huang R, et al. Survey of the translation shifts in hepatocellular carcinoma with ribosome profiling. Theranostics. 2019; 9: 4141-55. 